\title{
Case specificity of the stimulus probability effect
}

\author{
JEFF MILLER \\ University of California at San Diego, La Jolla, California 92093 \\ and \\ MARY HARDZINSKI \\ University of Michigan, Ann Arbor, Michigan 48104
}

\begin{abstract}
Four memory scanning experiments investigated the effect of the probability of occurrence of one case of a letter (e.g., "A") on response time to the other case of that same letter ("a"). There was no effect: Responses to one case of a letter did not depend on the probability of occurrence of the other case of the letter. This finding indicates that facilitation of visual encoding by high probability of occurrence is not caused by increased activation at the level of the name code. Previous results rule out the possibility that facilitation occurs at the level of feature detectors responding to the individual visual features of the high-probability stimuli, and these results were replicated in the present experiments. It appears that facilitation is caused by activation along specific routes by which visual features activate letter names. This conclusion has implications for the locus of effect of stimulus probability in models of letter coding.
\end{abstract}

The processes by which people identify letters have been the object of many experimental investigations over the last few years. An increasing body of experimental evidence is building in favor of letter recognition models of the sort proposed by Rumelhart (1970). In this model, physical information about the stimulus is assumed to be registered initially in a visual information store (VIS). From there, feature analysis processes extract information in order to recognize the letter, and once that has been accomplished, the name of the letter is transferred to short-term memory (STM). The letter name in STM is the representation that can be rehearsed, reported, or otherwise used in subsequent tasks. This sort of model accounts well for a variety of data obtained with tachistoscopic presentation of letter arrays, and similar models have been suggested by Massaro (1975), Neisser (1967), and Sperling (1967).

Models such as these are consistent with results from a variety of other paradigms, also indicating that visually presented letters are recognized on the basis of physical features and then remembered and dealt with on the basis of a name code. For example, studies of the retention of single letters in STM (e.g., Conrad, 1964; Wickelgren, 1965) have shown that the sorts of errors subjects make tend to be well predicted by an analysis

This research was supported in part by NSF Grant BNS7824772 to the first author. Experiments 1-3 were conducted in the laboratory of Robert G. Pachella, supported by NSF Grant BNS 76-82815, and we wish to express our appreciation for this assistance. We would also like to thank David Bauer and David Meyer for their incisive comments on earlier drafts of the paper. Requests for reprints should be addressed to Jeff Miller, Department of Psychology C-009, UCSD, La Jolla, California 92093. of acoustic similarities of the letters. Studies of letter matching by Posner and his colleagues are consistent with the same general view of letter perception. Their results support the idea that information about the physical characteristics of the letters becomes available first, followed by coding of the letter in terms of its name. Information about physical characteristics of the letters is generally used to make letter matching decisions only for about $1 \mathrm{sec}$ after the letter is presented, and after that the subject tends to rely entirely on the name code (e.g., Dainoff, 1970; Posner, Boies, Eichelman, \& Taylor, 1969; Posner \& Keele, 1967).

Evidence from the memory scanning task (Sternberg, 1969) also supports the idea that letters are coded by name before being compared with the memory set. Chase and Calfee (1969) and Siple (1975) found slower searching through acoustically similar positive sets than through acoustically nonconfusable sets, supporting an acoustic representation. Seamon and Wright (1976) showed that the memory set that is searched is closely tied to the memory set that is rehearsed subvocally. Hardzinski and Pachella (1980) and Wattenbarger (Note 1) attempted direct tests of the hypothesis that the output of the encoding stage is the name of the stimulus. Both of these studies employed upper- and lowercase letters in memory scanning tasks, with three experimental conditions. In the "name identity" condition, the upper-and lowercase versions of a given letter were always assigned to the same response, so that the subject merely had to identify stimulus letters by name. In the "physical identity" condition, upper- and lowercase versions were assigned to different responses, so the subject could use either a visual code for the stimulus or a name code elaborated to include case information 
as well. Finally, in a control condition, letters did not vary in case. In these experiments, the function relating reaction time (RT) to memory set size for the name identity conditions was nearly identical in both slope and intercept to those functions obtained for the control conditions, despite the fact that the name identity condition contained twice as many visual representations as did the control conditions. On the other hand, the slope for the function relating RT to set size for the physical identity condition was nearly twice as great as the slopes for the name identity and control conditions. This indicates that when subjects had to discriminate two letters having the same name, they had to use some more complicated representation than they normally use in the memory scanning task. Thus, it appears that the normal letter representation in this task is a case-independent name code.

Given the feature analysis to letter name conception of letter identification processes, it is important to understand what a name code is and how it becomes activated. Keele (1973) suggested that the logogen model of Morton (1969) could be applied to the area of letter recognition. According to this model, we have in our memories entities, called "logogens," corresponding to letter names. Logogens consist of generalizations of our knowledge about a particular letter, including information about different physical forms, different acoustic forms, position in the alphabet, and so on. These logogens can be activated by appropriate input from a variety of sensory modalities. When a logogen becomes activated above some threshold level, we become conscious of the letter name to which the logogen corresponds. An important property of the logogen system is that logogens can have differential activations below threshold and that the ease with which a given logogen can be activated above threshold by sensory input is dependent on its "resting" activation level. Experimental manipulations such as practice, number of response alternatives, stimulus repetition, differential payoffs for speed and accuracy (Keele, 1973), and the provision of advance information (Posner \& Snyder, 1975) may all have effects by changing the level of activation at a particular name code.

The idea of letter name codes as logogens is quite consistent with what is known about the effects of letter probability (frequency of presentation within an experimental block) on RT. In fact, there is some evidence to suggest that the probability effect is specifically caused by changes in the level of activation of a letter name code. First, Pachella and Miller (1976) found no probability effect in the letter matching task when subjects were not required to use the name of the stimulus letters. When subjects were asked to decide whether or not two letters were an exact physical match, probability had no effect, although it did have an effect when subjects were asked to decide whether or not two letters had the same name (cf. Besner, 1977).
A competing hypothesis, that probability affects the rate at which visual features are detected, is ruled out by the results of Miller (1979). ${ }^{1}$ He included pairs of visually similar letters such as " $E$ " and " $F$ " in a memory scanning task and found no influence of the probability of occurrence of one letter on RT to the other letter. Miller and Pachella (1973) showed that the effect of letter probability is larger when letters are presented in a visually degraded form than when they are presented under normal visual conditions. This interaction, obtained in both classification and naming tasks, is consistent with the hypothesis that both visual quality and letter probability affect the process of activating the name code for a letter, much as the logogen model suggests. Logogens corresponding to high-probability stimuli would have higher a priori activation levels, so less evidence from the visual system would be required to push the activation level above threshold. The probability effect arises because less evidence is needed to activate a high-probability stimulus than to activate a low-probability one. The interaction arises because the evidence is being accumulated at a higher rate when stimulus quality is good than when it is poor.

The idea that probability influences the time to activate a letter name seems to predict that a probability effect should be obtained in almost any task with letters used as stimuli. However, two studies using vocal naming responses have obtained no significant differences in RT as a function of letter probability (Hawkings, MacKay, Holley, Friedin, \& Cohen, 1973; Theios, Note 2), although other studies have obtained significant probability effects on naming RT (Miller \& Pachella, 1973; Stanovich \& Pachella, 1976). Two sources of artifact may account for these failures to find probability effects in the naming task. First (cf. Pachella, 1974; Stanovich \& Pachella, 1976), subjects often adopt very strict criteria for speed in naming tasks, thereby producing equal latencies across the various probability conditions but producing more errors on the lowprobability letters. Indeed, Stanovich and Pachella (1976) found that they could produce the probability effect in RTs by emphasizing accuracy in the instructions to the subjects or produce the effect in accuracy by emphasizing speed. As Pachella (1974) noted, the error rates in the Theios (Note 2) study are remarkably consistent with the idea that there was an effect of probability on accuracy. Second, probability has not always been varied over the same range. The effect of probability on RT is typically found to be quite small unless very low-probability stimuli ( $<$.05) are used (cf. Miller \& Pachella, 1973; Theios, Smith, Haviland, Traupmann, \& Moy, 1973). However, the Hawkins et al. study compared probabilities of .5 and .1. It is therefore easy to explain their nonsignificant probability effect in terms of a lack of power, since the effect is normally very small in this rarige anyway.

The nonlinearity of the probability effect may also explain another finding embarrassing to the hypothesis 
that probability influences the speed of activating a name code. Hardzinski and Pachella (1980) and Sternberg (1969) reported additivity of visual quality and set size in the memory scanning task. Since items in the larger set sizes were tested less frequently than items in the smaller set sizes, it seems that a probability effect should have produced a Quality by Set Size interaction. However, the variation in stimulus probability may not have been sufficient to produce this interaction. Sternberg's positive-set items varied in probability only from .267 to .067 , a range that does not usually produce either a large probability effect or a large Probability by Quality interaction. The probability range in Hardzinski and Pachella was even more restricted, from .25 to .10 . The question of why probability seems to have so much larger effects in the range of small probabilities is an interesting one, but beyond the scope of this paper.

The purpose of the present experiments is to investigate the theory that probability affects the time needed to activate a name code during the encoding of alphanumeric characters in the memory scanning task. According to the model just outlined, different visual stimuli having a common name should both contribute to and should both benefit from a buildup of excitation in their common name code. The present experiments use both upper- and lowercase versions of stimulus letters in a memory scanning task to test this prediction. In all experiments, the response is the same for different cases of a given letter. For each letter, however, the probability of occurrence of one case of the letter is chosen from a widely varying set of values, whereas the probability of occurrence of the other case is assigned a standard value. According to the model proposed to account for probability effects, an effect due to the frequency of occurrence of an uppercase letter should transfer to the lowercase letter with the same name, and vice versa. Furthermore, the size of the effect of stimulus probability should depend on stimulus quality not only for an uppercase letter whose frequency is being manipulated directly, but also for the lowercase version of that letter. Thus, the common logogen interpretation predicts both that probability effects should transfer from one case of a letter to another and that the size of the transfer should depend on the quality of the stimulus. Both of these predictions follow directly from the part of the model that says that the effect of probability is to change the excitation level of the name code common to the two versions of a single letter.

In these experiments, two methods of degradation were used: contrast reduction and superimposition of a random dot pattern over the stimulus. Turvey (1973) has shown that different methods of reducing visual quality may have effects at different levels within the visual system and that differences in the effects of visual quality manipulations may reveal subprocesses within visual encoding. On the basis of Turvey's results, contrast may be expected to have an effect at a somewhat lower level within the visual system than do random dot patterns. If so, then these two methods of degradation may produce different interactions with probability (cf. Miller, 1979).

\section{EXPERIMENT 1}

\section{Method}

Subjects. Twenty-four right-handed paid volunteers were recruited on the University of Michigan campus to serve as subjects in the experiment.

Apparatus. A PDP-1 computer controlled the presentation of stimuli, recorded responses and response latencies, and provided the subject with feedback about correct and incorrect responses. Stimuli were presented on an HP 1130A cathode-ray tube (CRT) located approximately $65 \mathrm{~cm}$ from the subject. The stimuli were upper- and lowercase consonants (B, D, F, H, $M, N, R, T$ ) drawn in a standard, highly recognizable type font. The uppercase letters were $1.2 \mathrm{~cm}$ high. The lowercase letters varied from $1.2 \mathrm{~cm}$ (e.g., "f") to $.6 \mathrm{~cm}$ (e.g., "m") high. The letters subtended visual angles of 1.0 and $.5 \mathrm{deg}$. All letters were $.7 \mathrm{~cm}$ wide. Positive and negative responses were made with the right and left index fingers, respectively, via a microswitch keyboard located directly in front of the subject.

Design and Procedure. Subjects were run in one 1-h session. A session consisted of one practice block of 200 trials, followed by three experimental blocks of 400 trials each. The three experimental blocks differed according to the visual conditions under which the stimulus was presented. For the normal block, stimuli were presented without any visual degradation. For the lowcontrast blocks, a filter was placed over the display. The filter reduced the intensity of the stimulus against its background by approximately $1.5 \log$ units. In the visual noise blocks, a random pattern of dots, varying from trial to trial, was superimposed over the test stimulus. Dot patterns were composed of 144 dots scattered over an area $3 \mathrm{~cm}$ on a side. The patterns were generated by treating the area as a 12 by 12 grid of smaller squares. On each trial, one dot was randomly placed within each of the small square areas of the grid. The order of testing of the three visual quality conditions was counterbalanced across subjects.

Half of the stimulus set was designated as the positive set for a subject at the beginning of the session. The positive set included eight stimuli: the upper- and lowercase representations of four different letters. Subjects were shown all 16 letters at the beginning of a session, but they were not told specifically of the negative set or the probability manipulation. The assignment of letters to the positive and negative sets was randomized across subjects such that no subject received the same positive or negative set as another subject. Half the trials within a block required positive responses and half, negative.

For each of the four letter names in the positive set and each of the four in the negative set, one case of the letter was assigned to the direct probability manipulation condition and the other case of the letter was assigned to the transfer conditions (see Table 1). Letters in the direct probability manipulation condition occurred with probabilities of $.2125, .1525, .0725$, and .0125 . The other cases of these letters in the transfer condition all occurred with probabilities of .0125 . In order to minimize the informational value of the case of the stimulus with respect to the correct response, these assignments were made so that the highest and lowest probability items would be of one case (i.e., both uppercase or both lowercase) and the two middle probability items would be of the other case. Thus upperand lowercase letters occurred equally often within a given response type and across positive and negative responses. Assignments of letters and cases to probability conditions were counterbalanced across subjects.

A trial began with the presentation of a test stimulus. The stimulus remained on the screen until the subject responded or 
Table 1

Sample Stimulus Set for Experiment 1

\begin{tabular}{|c|c|c|c|c|c|c|c|}
\hline \multicolumn{4}{|c|}{ Positive Set } & \multicolumn{4}{|c|}{ Negative Set } \\
\hline \multicolumn{2}{|c|}{ Direct } & \multicolumn{2}{|c|}{ Transfer } & \multicolumn{2}{|c|}{ Direct } & \multicolumn{2}{|c|}{ Transfer } \\
\hline $\mathbf{L}$ & $\mathbf{P}$ & $\mathbf{L}$ & $\mathbf{P}$ & $\mathrm{L}$ & $\mathbf{P}$ & $L$ & $\mathbf{P}$ \\
\hline B & .0125 & $\mathbf{b}$ & .0125 & $\mathrm{~m}$ & .0125 & $\mathbf{M}$ & .0125 \\
\hline d & .0725 & $\mathrm{D}$ & .0125 & $\mathrm{~N}$ & .0725 & $\mathrm{n}$ & .0125 \\
\hline f & .1525 & $F$ & .0125 & $\mathbf{R}$ & .1525 & $\mathrm{I}$ & .0125 \\
\hline H & .2125 & $\mathrm{~h}$ & .0125 & $t$ & .2125 & $\mathbf{T}$ & .0125 \\
\hline
\end{tabular}

Note $-L=$ letter $P=$ probability.

$3 \mathrm{sec}$ had elapsed. Following the response, the subject was given feedback in the form of a "+" for correct responses and a "-" for incorrect responses. The feedback remained on the screen for $500 \mathrm{msec}$ and was followed by a $500-\mathrm{msec}$ intertrial interval. Error trials and trials that exceeded the 3-sec criterion time were recorded but otherwise ignored in the analysis. These trials were repeated later in the block.

\section{Results and Discussion}

For each block of trials, two groups of eight average RTs and percentages of error were computed: one group for stimuli in the direct probability manipulation condition and one group for stimuli in the transfer condition. For each group, there were four stimuli in the positive set and four in the negative set, and these four stimuli were assigned to different levels of probability. Average values across subjects and positive vs. negative set are shown in Figures 1 and 2, as a function of stimulus probability and visual conditions of presentation. Separate analyses of variance were performed on the results of the direct and transfer conditions. In each analysis, the factors were visual conditions, response type, probability level, and subjects. Results from the direct condition were straightforward. The main effects of visual condition $[\mathrm{F}(2,46)=51, \mathrm{p}<.01, \mathrm{MSe}=19,506]$, response type $[F(1,23)=12, p<.01, \mathrm{MSe}=10,967]$, and probability $[\mathrm{F}(3,69)=40, \mathrm{p}<.01, \mathrm{MSe}=6,067]$ were all highly significant. The main effects of visual condition and probability are apparent in Figure 1, and the effect of response type was a 30-msec advantage for the positive response. The interaction of visual condition and probability, shown in Figure 1, was also highly significant $[F(6,138)=5.3, p<.01, \mathrm{MSe}=4,004]$, and no other interactions were significant. This interaction was due to a larger probability effect in the presence of visual noise than in either the normal or low-contrast condition. The effect of probability was not larger in the low-contrast condition than in the normal condition $(F<1, p>.20)$.

The results from the indirect condition were slightly more complicated. The main effects of visual condition $[F(2,46)=34, p<.01, \mathrm{MSe}=50,731]$ and response type $[F(1,23)=10, p<.01, \quad M S e=15,266]$ were significant, but the effect of probability $[\mathrm{F}(3,69)=1.9$, $p>.10, \mathrm{MSe}=16,498]$ was not. The response type effect was a 34-msec advantage for the positive response.
Two interactions were barely significant in this analysis: the two-way interaction of visual condition and response type $[F(2,46)=3.4, p<.05, \mathrm{MSe}=6,745]$ and the three-way interaction of visual condition, response type, and probability $[\mathrm{F}(6,138)=2.4, \mathrm{p}<.05, \mathrm{MSe}=9,900]$.

In order to see whether the Probability by Quality interaction was significantly larger in the direct condition than in the transfer condition, a further analysis was done using data from both conditions. The three-way interaction of probability, quality, and direct vs. transfer condition was marginally significant $[F(6,138)=1.93$, $\mathrm{p}<.10, \mathrm{MSe}=5,690]$. Looking only at the data from the normal and visual noise conditions, the three-way interaction was significant $[\mathrm{F}(3,69)=2.9, \mathrm{p}<.05$. $\mathrm{MSe}=7,193$ ] .

Results from the analyses of error rates did not suggest that any of the RT findings were due to speedaccuracy tradeoffs. In the analysis of the direct condi-

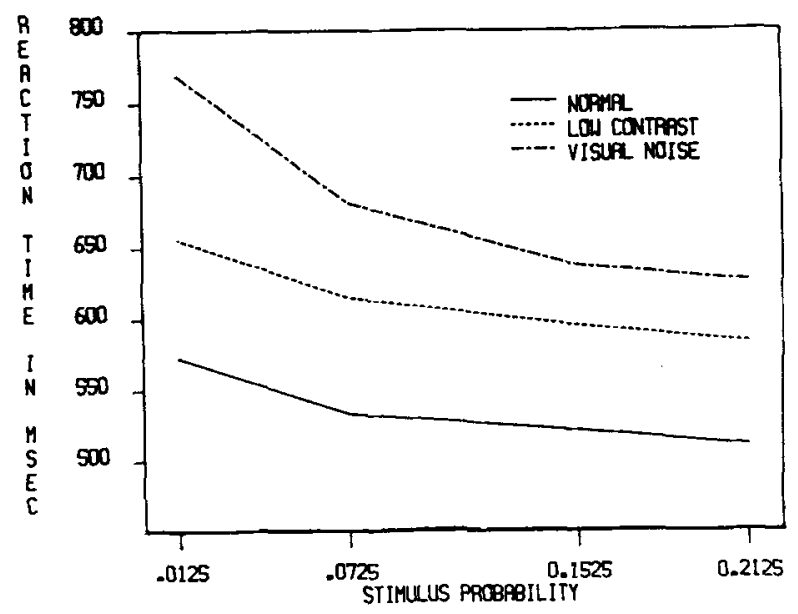

Figure 1. Reaction time as a function of stimulus probability and visual condition for the direct probability condition of Experiment 1.

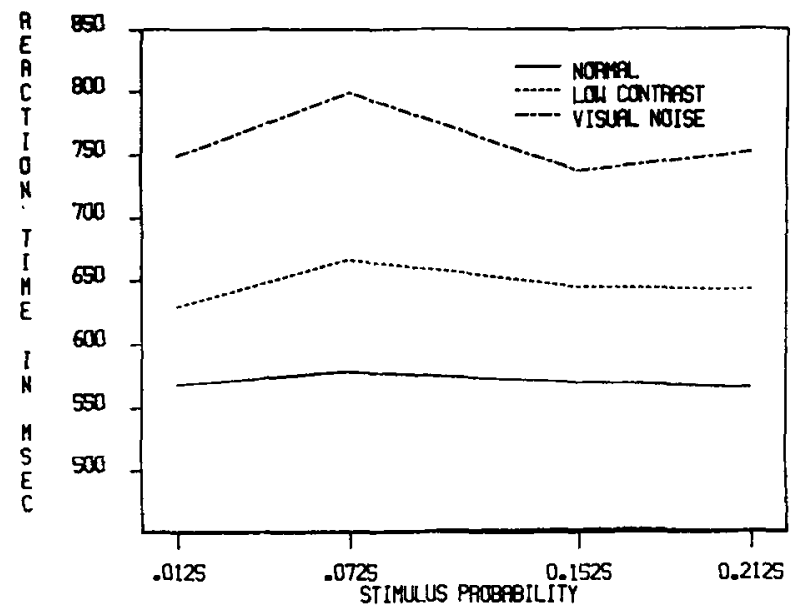

Figure 2. Reaction time as a function of stimulus probability and visual condition for the transfer condition of Experiment 1. 
tion, the only significant source of variance was the main effect of probability $[F(3,69)=6.1, p<.01$, $\mathrm{MSe}=1,766]$, with errors increasing as probability decreased. There were no significant sources of variance in the analysis of error rates in the indirect condition.

The results of the conditions in which probability was manipulated directly demonstrate an effect of stimulus probability on RT in the memory scanning task and an interaction of stimulus probability with stimulus quality. Both results have previously been reported by Miller and Pachella $(1973,1976)$. Data from the present study extend the previous findings by demonstrating an interaction of probability with visual noise produced by superimposing random dot patterns over the stimulus, since earlier studies used contrast reduction as the only means of degrading the stimulus. The interaction of probability with visual noise in these data was strong and highly significant. Failure to replicate the interaction of probability with contrast reduction may have been due to a slightly smaller contrast effect than is usually found in studies obtaining the interaction, or it may simply have been a Type II error. The interaction was replicated in Experiments 2 and 3.

The results from the transfer condition contradict the predictions derived from the logogen model outlined earlier. Not only was there no interaction of probability with visual quality in these data, but there was also no main effect of probability. Averaged across the range of probabilities used in this experiment, there was a decrease of only $6 \mathrm{msec}$ in RT for every .1 increase in stimulus probability for the transfer condition. Furthermore, $95 \%$ confidence limits for the size of this decrease were -8 to $20 \mathrm{msec}$. In contrast, there was a $46-\mathrm{sec}$ decrease in RT for every .1 increase in probability in the direct probability condition, with $95 \%$ confidence limits of 32 to $60 \mathrm{msec}$. Thus, there was little or no transfer of the probability effect from one case of a letter, for which probability was manipulated, to the other case of that letter, for which probability was not manipulated.

It is possible to argue, in spite of the evidence presented in the introduction, that separate logogens are used for different cases of a letter. If so, then the prob. ability effect would not be expected to transfer from one case of a letter to another, and the failure to find transfer could not be regarded as evidence against the hypothesis that probability affects the level of activation in letter logogens. Thus, it seemed important to look for converging evidence within this task suggesting that different cases of a letter activate the same logogen. In order to get some evidence relevant to this point, sequential effects were examined. Each trial was reclassified according to whether it was (1) a repetition of the same letter in the same case as the previous trial, (2) a repetition of the same letter in the case opposite from the previous trial, (3) a different letter from the previous trial, but a letter requiring the same response, or (4) a different letter requiring the opposite response. The mean RTs for responses in these four repetition classes were $530,575,603$, and $597 \mathrm{msec}$, respectively. All pairs of these means are reliably different $(p<.01)$ by Tukey's test, except for the pair consisting of Classes 3 and 4 . This pattern was obtained for letters in each of the three highest probability levels, although there were too few repetitions for an analysis of the .0125 stimuli. The finding that repetitions of a given letter were faster than nonrepetitions even when the case of the letter changed shows that processing of the two cases of a letter is not independent and provides further evidence that they share a common name code. Combined with the absence of transfer of the probability effect, this finding indicates that the repetition effect is mediated by a mechanism somewhat different from that which mediates the probability effect.

The interactions of visual quality with response type and of visual quality, response type, and probability in the transfer condition are somewhat difficult to explain. The means involved in these interactions are shown in Table 2. It appears that the difference between positive and negative response times was greater for high-probability items than for low-probability items in the normal and low-contrast conditions, but that this was reversed in the condition with degradation by superimposed random dot patterns. One ad hoc explanation of this finding involves the notion of an initial familiarity check proposed by Atkinson and Juola (1973). It could be that in the normal and low-contrast conditions, familiarity of the item tends to favor a positive response. When a high-probability item from the negative set is presented, the high familiarity favors the positive response, thus tending to delay the negative response. In the condition with visual noise, this familiarity response may be suppressed by the increased difficulty of the task or by the varying nature of the superimposed random dots, thus eliminating the competition with the appropriate response.

\section{EXPERIMENT 2}

The conclusion that there is no transfer of the prob. ability effect from one case of a letter to another case of the same letter should probably be made cautiously, since it is based on an acceptance of a null hypothesis. For this reason, Experiment 2 was designed to replicate Experiment 1 with a slight change in procedure. In

Table 2

\begin{tabular}{llcccc}
\hline \multirow{2}{*}{$\begin{array}{c}\text { Visual } \\
\text { Condition }\end{array}$} & $\begin{array}{c}\text { Response } \\
\text { Type }\end{array}$ & \multicolumn{4}{c}{ Stimulus Probability } \\
\cline { 3 - 6 } Normal & Positive & 547 & 542 & 582 & 593 \\
& Negative & 585 & 599 & 574 & 542 \\
Low Contrast & Positive & 600 & 605 & 648 & 629 \\
& Negative & 687 & 685 & 684 & 629 \\
Visual Noise & Positive & 747 & 731 & 761 & 717 \\
& Negative & 756 & 744 & 838 & 782 \\
\hline
\end{tabular}


Experiment 2, transfer was examined between different blocks of experimental trials. Only eight stimulus forms were used in a given block, and all were of the same case. In successive blocks, however, different cases were used, and the transfer of probability effects from one block to the next was examined.

\section{Method}

Subjects. Twenty-five right-handed paid volunteers were recruited on the University of Michigan campus to serve as subjects in the experiment.

Apparatus and Procedure. The same apparatus was used for data collection as that used in Experiment 1. The stimuli were also the same, except that the letter " $K$ " replaced the letter " $R$ ", to increase stimulus discriminability. Certain variations in procedure were required in order to have the direct and transfer conditions in different blocks of trials. A session consisted of one practice block of 120 trials followed by three pairs of experimental blocks. Within each pair of blocks, the first block was the block in which probability was manipulated directly. There were a total of 240 trials in this block. The four stimuli in the positive and negative sets occurred with frequencies of $67,30,18$, and 5 . In the direct probability manipulation block, either all stimuli were uppercase letters or all were lowercase (counterbalanced across subjects). The second block within each pair consisted of only 120 trials, and the stimulus letters were the opposite-case versions of the letters that were used in the longer blocks. In these blocks, all eight stimuli occurred with equal frequency. The pairs of experimental blocks differed according to the type of visual degradation imposed on the test stimulus, with the same visual conditions used in Experiment 1.

\section{Results and Discussion}

Any subject who made more than $8 \%$ errors in any condition was replaced with a different subject. One subject was excluded by this criterion. Average RTs and percentages of error were computed for each of the eight stimuli in any given block of trials for each remaining subject. These values were averaged across subjects and positive vs. negative set, and the averages are displayed in Figures 3 and 4, as a function of probability, visual condition, and direct vs. transfer condition.

Separate analyses of variance were performed on the results from the direct probability manipulation blocks and the transfer blocks, with factors of visual conditions, probability, response type, and subject. In the direct condition, the main effects of visual conditions $[\mathrm{F}(2,46)=30.5, \mathrm{p}<.01, \mathrm{MSe}=26,242]$, response type $[\mathrm{F}(1,23)=20.4, \mathrm{p}<.01, \mathrm{MSe}=6,346]$, and probability $[\mathrm{F}(3,69)=36.8, \mathrm{p}<.01, \mathrm{MSe}=8,784]$ were all significant. The only significant interaction was that of probability with visual conditions $[F(6,138)=3.72$, $\mathrm{p}<.01, \mathrm{MSe}=3,904]$. The effect of response type was a 30-msec advantage for the positive response, and the other effects are apparent in Figure 3. A similar analysis of variance of the error rates in the direct probability manipulation condition yielded a reliable main effect of probability $[\mathrm{F}(3,69)=6.73, \mathrm{p}<.01, \mathrm{MSe}=59.5]$, with error rates increasing as stimulus probability decreased. Analysis of the latency data from the transfer blocks

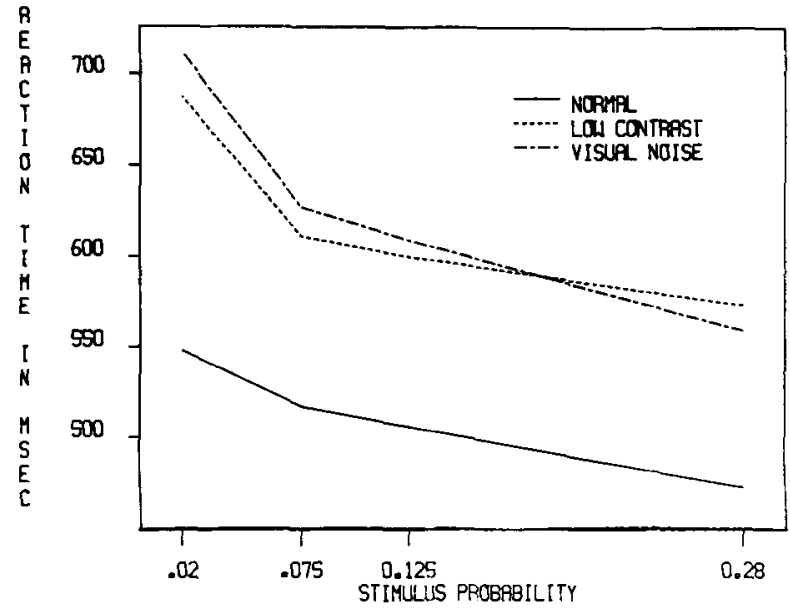

Figure 3. Reaction time as a function of stimulus probability and visual condition for the direct probability condition of Experiment 2.

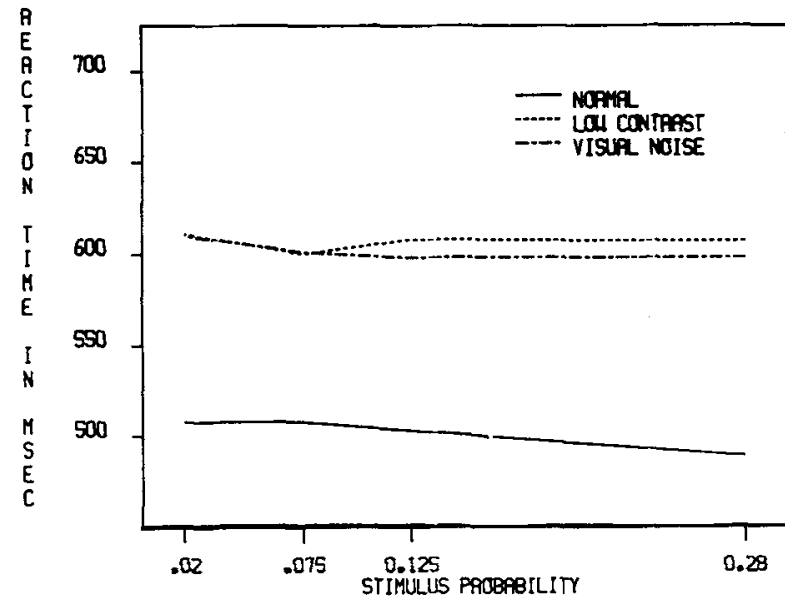

Figure 4. Reaction time as a function of stimulus probability and visual condition for the transfer condition of Experiment 2.

indicated significant main effects of visual conditions $[\mathrm{F}(2,46)=47.4, \mathrm{p}<.01, \mathrm{MSe}=14,114]$ and response type $[F(1,23)=19.5, p<.01, \mathrm{MSe}=7,988]$. Neither the effect of probability $[F(3,69)=.83, p>.10, \mathrm{MSe}=$ $3,619]$ nor the interaction of probability with visual quality $[\mathrm{F}(6,138)=1.01, \mathrm{p}>.10, \mathrm{MSe}=1,603]$ was significant. The only significant effect in the error rates was the main effect of visual conditions $[\mathrm{F}(2,46)=6.65$, $\mathrm{p}<.01, \mathrm{MSe}=16.5]$.

As in Experiment 1, a further analysis was performed with data from both the direct and transfer conditions. The Probability by Quality interaction was reliably larger in the direct condition than in the transfer condition $[F(6,138)=3.23, p<.01, \mathrm{MSe}=2,405]$.

The results of Experiment 2 are completely consistent with the results of Experiment 1 with respect to the lack of transfer of the probability effect between upper- and lowercase versions of a single letter. As in 
Experiment 1, 95\% confidence limits were computed for the decrease in RT produced by an increase in stimulus probability of .1. These limits were 31.5 to 45.5 for stimuli whose probabilities were manipulated directly, but only -3.5 to 10.5 for the transfer stimuli. Thus, the evidence again suggests that there is no transfer. The extension of this result to a task with transfer across rather than within blocks adds generality to the finding and leaves little doubt that the facilitation produced by high frequency of occurrence of a given item does not generalize to other stimuli with the same name.

\section{EXPERIMENT 3}

One possible criticism of Experiment 2 could be based on the idea that the effects of probability may be extremely transitory. There is some evidence that the effects of probability carry over from one block of trials to the next. Geller, Whitman, and Post (1973) presented stimuli with one set of probabilities initially and then reversed probabilities in a subsequent block. There appeared to be a residual effect of the probabilities in the initial block, even when subjects were informed of the probability reversals. On the other hand, the encoding mechanisms sensitive to the effects of probability might respond very quickly to changes in stimulus probabilities. If so, then the present experiment might not detect a carryover from one set of stimuli to another set with the same names but different cases. The probability effect might vanish so quickly in the equal-probability blocks that no effect could be detected. In order to examine this possibility, a third experiment was conducted. In this experiment, the probabilities were manipulated just as in the second experiment, but the letters in the equal-probability blocks were identical to the letters in the unequalprobability blocks, instead of being different in case.

\section{Method}

Twenty-eight right-handed paid volunteers were recruited on the University of Michigan campus to serve as subjects in this experiment. The details of the apparatus and procedure were nearly identical to those of Experiment 2, except that in this experiment all of the letters in both types of blocks were of a single case. In other words, the blocks in which probability was manipulated directly and the transfer blocks used identical letters. The case of letters used was counterbalanced across subjects.

\section{Results and Discussion}

The procedure for analyzing these data was identical to the procedure used in the analysis of Experiment 2. Four subjects were excluded for exceeding the criterion of $8 \%$ errors in any condition. Figures 5 and 6 show the averages, across subjects and response types, for letters in the different probability and visual conditions, both for letters in the direct probability blocks and the transfer blocks. Separate analyses of variance were performed for data from the direct and transfer blocks, as in the earlier experiments. Results from the blocks in which probability was actually manipulated replicated earlier findings. The main effects of visual conditions $[\mathrm{F}(2,46)=56, \mathrm{p}<.01, \mathrm{MSe}=15,743]$ and probability $[F(3,69)=27, p<.01, \mathrm{MSe}=7,742]$ were both significant, as was their interaction $[F(6,138)=2.58$, $p<.025, \mathrm{MSe}=2,848]$. The analysis of the transfer blocks showed a somewhat different pattern of results. The effect of visual conditions $[F(2,46)=47.4, p<.01$, MSe $=14,114]$ was comparable to that obtained in the direct probability manipulation blocks. However, results from the transfer blocks showed a much smaller effect of probability $[\mathrm{F}(3,69)=6.0, \mathrm{p}<.01$, MSe $=$ 4,093 ] and no interaction of visual quality with probability $[F(6,138)=.86, p>.10, \mathrm{MSe}=1,572]$.

Analyses of the error rates showed that subjects tended to make more errors in the conditions in which their responses were slower. In the direct probability

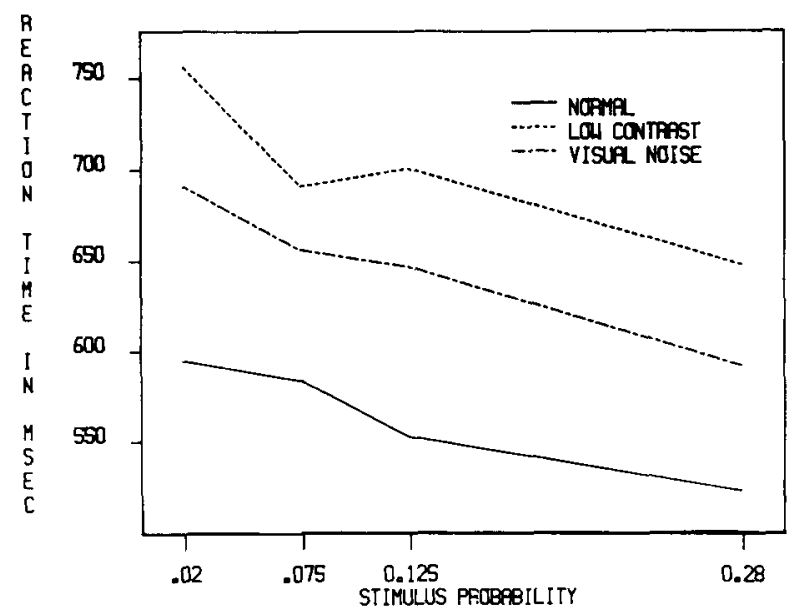

Figure 5. Reaction time as a function of stimulus probability and visual condition for the direct probability condition of Experiment 3.

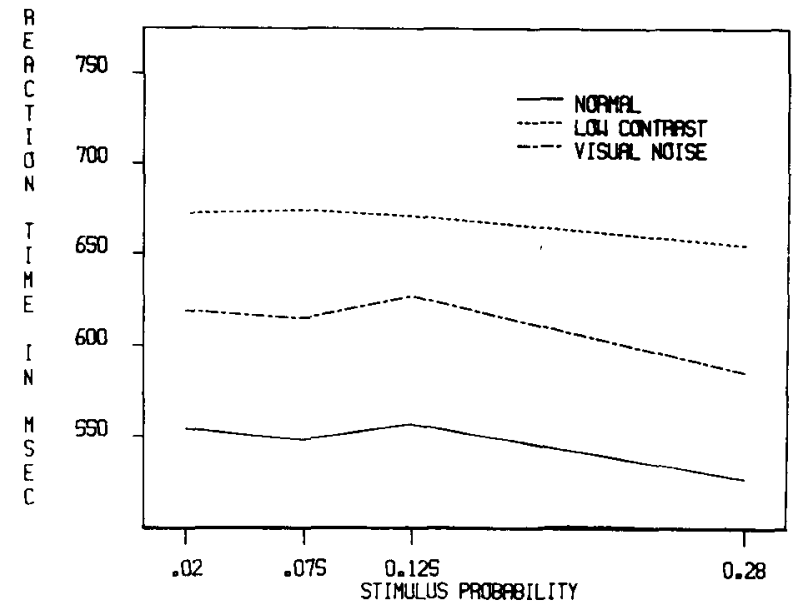

Figure 6. Reaction time as a function of stimulus probability and visual condition for the transfer condition of Experiment 3. 
manipulation blocks, error rates were higher for lowprobability stimuli $[\mathrm{F}(3,69)=6.7, \mathrm{p}<.01, \mathrm{MSe}=59.5]$, whereas in the transfer blocks, error rates were slightly but consistently higher $[\mathrm{F}(2,46)=6.6, \mathrm{p}<.01$, MSe $=$ $16.5]$ in the visually degraded conditions (about $3 \%$ ) than in the normal visual condition (about $2 \%$ ).

These results indicate that the effect of probability is somewhat transitory, at least in the context of the experimental design used here. The decrease in RT associated with a .1 increase in probability was somewhere between 28 and $39 \mathrm{msec}$ in the direct condition, but only $4.5-15.5 \mathrm{msec}$ in the transfer block. However, the results support the view that the transfer design is sufficiently sensitive to detect even small probability effects, so the lack of a transfer effect in Experiment 2 cannot be attributed solely to lack of power.

\section{EXPERIMENT 4}

The results of the first three experiments suggest that the facilitation of response to a high-probability letter may be specific to the exact form of the letter that occurs with high probability, since there was no transfer of the probability effect across cases. This finding contradicts the simple notion that probability affects the amount of visual evidence required to activate the name of a letter. Within the framework of traditional models of letter recognition, these results cast doubt on the assumptions that name codes may have different activation levels and that activation levels are sensitive to experimental manipulations. It seems that, at least for manipulations of stimulus probability within experimental blocks, activation levels of different name codes do not respond to presentation probabilities.

Given that the transfer paradigm indicates no effect of probability on the level of activation either of name codes or of visual features, it seems reasonable to ask whether the probability effect ever transfers. Miller (1969, Experiment 4) obtained transfer with geometric figures constructed from two binary attributes $(X$ vs. diamond shape, with bar across top of bottom of figure). He argued that the two stimulus attributes were perceived and coded separately, however, so transfer was caused by identical stimulus features activating identical codes. If so, that experiment did not really indicate transfer to a nonidentical stimulus. In the present experiment, we examined transfer of the probability effect from a high-probability stimulus to another stimulus identical to it except for size. Many theories of letter recognition are based on the idea of letter features that are at least partly independent of size (e.g., Neisser, 1967). If the probability effect transfers when two stimuli with common features activate the same code, then there should be a transfer of the probability effect from a high-probability stimulus to another stimulus differing only in size.
For comparison purposes, several other types of stimuli were included. First, transfer to visually similar stimuli with different names was examined, in order to replicate and extend the findings of Miller (1979). Second, transfer to different-case letters was also included, as a further replication of the results of Experiments 1 and 2. Third, transfer to same-case versions of letters in a different type font was examined. Strong versions of the hypothesis of case-specific letter logogens predict transfer to same-case versions of a letter, even if the letter is in a different type font.

\section{Method}

Each of 24 right-handed subjects was run in two blocks of 200 trials. Subjects performed a memory scanning task, with fixed positive and negative sets of Size 3 . Stimuli were presented and responses and response latencies were recorded by a Terak microcomputer. Stimuli were presented on a CRT screen, using a high-resolution bit-map display. Subjects viewed the screen from a distance of about $65 \mathrm{~cm}$ and made responses with the left and right index fingers by pressing one of two keys on the computer keyboard. The general structure of a trial and the presentation of feedback were similar to the earlier experiments.

The stimulus set used in this experiment is shown in Figure 7. The eight stimulus letters were $B, D, I, T, O, Q, P$, and $R$, and each letter appeared in four different forms. The basic form of the letter was that of the Rumelhart (Note 3) font, shown in the leftmost column. The letters were selected from this font so that for each letter in the set, there would be exactly one letter differing from it by only one visual feature. Defining visually similar letters as those that differ by only one visual feature, then, these stimulus letters form four pairs of visually similar letters. In addition to the basic Rumelhart font, a second type font was constructed to be quite different from the Rumelhart font. $^{2}$ This font is shown in the second column in Figure 7, and it will be referred to as the "triangular" font. The third form of each letter was a smaller version of the Rumelhart font. Heights and widths of the smaller letters were $40 \%$ of the values used for the full-size letters. Finally, a lowercase version of each letter was used.

Six of the eight letter names were used for each subject, with three letter names assigned to the positive response and three letter names assigned to the negative response. Subjects were shown the stimulus set in advance, and they were instructed to

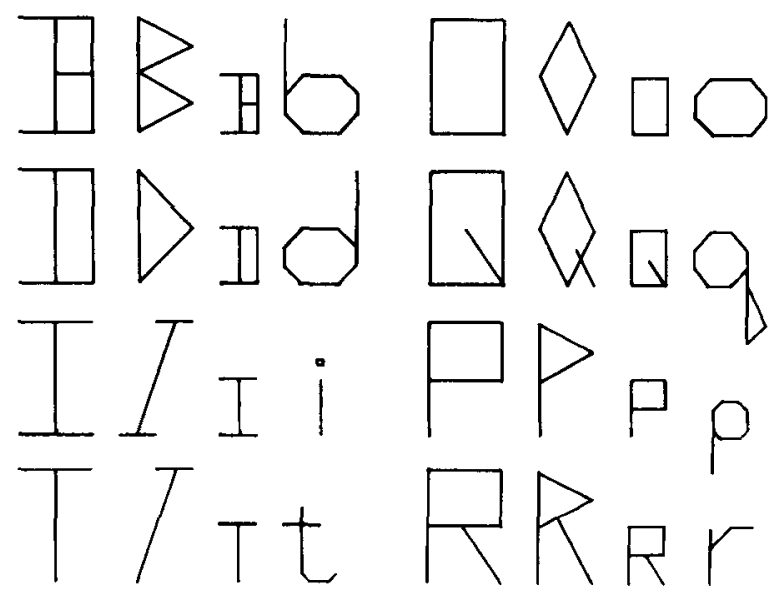

Figure 7. Stimulus set for Experiment 4. 
respond on the basis of the name of the stimulus letter, ignoring differences in font. Of the three letter names assigned to each response, one was designated the high-probability letter. For this letter, the large version in the Rumelhart (Note 3) font appeared on $33 \%$ of all trials. All other versions of this letter appeared on $1.5 \%$ of all trials. A second letter assigned to each response was the letter whose version in the Rumelhart font was most similar to the high-probability letter in the Rumelhart font. Each version of this letter appeared on $1.5 \%$ of the trials. Finally, the third letter assigned to each response was dissimilar to all other letters used for that subject, and each version of this letter appeared on $1.5 \%$ of the trials. The positive and negative sets given to the first subject, for example, were BDI and QOR. For this subject, the large Rumelhart B and the large Rumelhart $Q$ each appeared on $33 \%$ of the trials. The other 22 visual forms of these six letters each appeared on $1.5 \%$ of the trials, and the letters $T$ and $P$ were not used for this subject. The assignment of letters to probability conditions was counterbalanced across subjects.

\section{Results}

For each subject and each stimulus, average RT and percentage of correct response were computed for each stimulus and each block. Averages of these values across subjects, blocks, and letters are shown in Figure 8. Although the only visual forms that actually occurred with high probability were the letters in the large Rumelhart (Note 3) font, other-font letters with the same name are also shown on the high-probability curve. An analysis of variance on the latency data indicated significant differences among the probability conditions $[\mathrm{F}(2,46)=13, \mathrm{p}<.01, \mathrm{MSe}=26,534]$ and among the fonts $[\mathrm{F}(3,69)=17.5, \mathrm{p}<.01, \mathrm{MSe}=13,299]$. In addition, the interaction of these two factors was significant $[F(6,138)=4.53, p<.01, \mathrm{MSe}=15,553]$. An analysis of variance comparing only the physically similar letter and the physically dissimilar letter indicated that neither the difference between these two letters nor the interaction of these letter types with font was significant (both Fs $<1$ ). Post hoc analysis using the Newman-Keuls procedure for pairwise comparisons

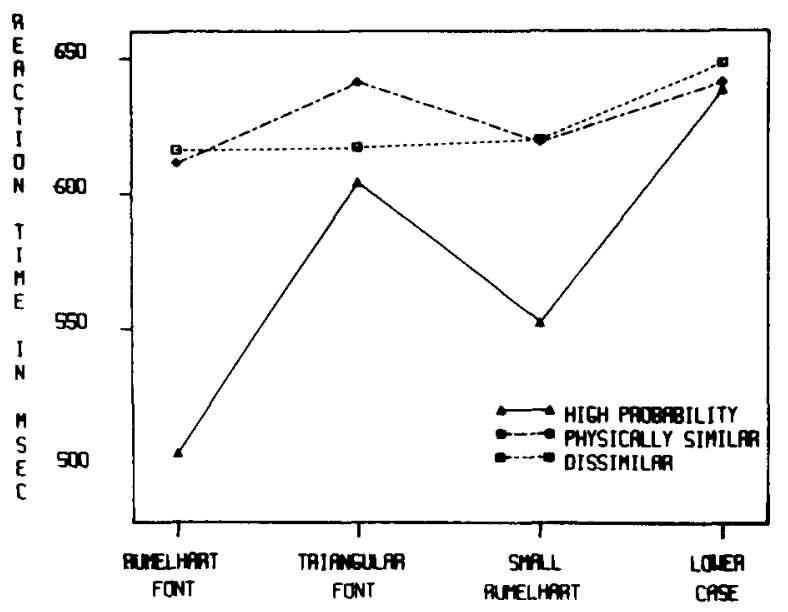

Figure 8. Reaction time as a function of stimulus type and probability condition in Experiment 4. Only letters in the large Rumelhart font actually occurred with high probability. confirmed that the high-probability Rumelhart letter and its small-size version were significantly $(p<.05)$ different from each other and from the rest of the letters. Differences among the other points were not significant, however. Thus there was a significant probability effect, and this effect transferred to the same form reduced in size. However, there was no transfer to the visually similar letter, the lowercase version of the letter, or the same-case version in the triangular type font.

The inclusion of tests for transfer to visually similar, different-case, different-font, and different-size items all in the same experiment allows two alternative explanations of earlier results to be discounted. First, one could argue that case-specific physical rather than name coding was used in Experiments 1 and 2, in spite of the evidence against this possibility. This argument is even more implausible in this experiment, however. The fact that there were 24 different visual forms to deal with, but only six letter names, would surely encourage subjects to use name coding to simplify the demands on memory. Also, the use of physical coding would surely produce transfer to the visually similar stimulus, but no such transfer was obtained. This lack of transfer is particularly dramatic because the similar stimulus was always assigned to the same response as was the highprobability stimulus, making it as easy as possible for subjects to use physical codes. Second, the argument that the transfer paradigm has insufficient power is weakened by the results of this experiment. Clear-cut transfer was obtained for one condition, strengthening the conclusion that there is no transfer for the other conditions.

\section{GENERAL DISCUSSION}

The results of these studies help identify the stimuli that benefit when a letter is presented frequently. The exact high-probability stimulus itself of course benefits, and a different size of the same visual form can also benefit. On the other hand, there seems to be no transfer of the probability effect to other stimuli with the same name as the high-probability stimulus. Neither a lowercase version of the high-probability letter nor an uppercase version from a very different type font will benefit when an uppercase letter is presented with high probability.

The finding of no transfer to lowercase and differentfont versions of a letter is important because it shows that the probability effect is tied to the particular visual features presented frequently. On the other hand, the fact that there is no transfer to visually similar letters having a different name (Experiment 4; Miller, 1979) demonstrates that probability does not simply activate feature detectors for the high-probability visual features.

What are the implications of these results for models of the type outlined in the introduction? While previous 
findings indicated that the probability effect seems to arise in the process of activating a name code for the stimulus, the present results indicate that the effect is specific to one particular mode of activating this name code. We will refer to this idea as "route-specific" activation, and we propose to model it within a logogen framework. It is possible to conceive of facilitation as a property of the path or route by which evidence of the high-probability features is sent to the appropriate logogen. For example, we might imagine that the evidence for the presence of a horizontal line in the letter " $\mathrm{A}$ " is more quickly sent to the logogen corresponding to the name "a" when the visual form " $\mathrm{A}$ " is a high-probability stimulus than when it is a lowprobability stimulus. This transmission rate could be higher even in a system for which neither the speed of detecting the horizontal line nor the amount of evidence required to identify an "a" is affected by probability. We suggest that the facilitation due to high frequency of occurrence is neither in the process of extracting the visual features nor in the process of activating the appropriate letter-name logogen, but rather in the transmission of evidence concerning visual features to that logogen.

According to this modified logogen model, the transfer to different-size stimulus letters is based on partial overlap of the route by which visual information activates the letter name. It is remarkable that only $50 \%$ of the probability effect transferred to a stimulus differing only in size. To the extent that the transfer was not perfect, we may conclude that there is some separation of the channels through which visual information about the large and small letters is processed. One possibility, for example, is that processing is separate up to the level of a mechanism for size normalization, after which the same route is used.

As an alternative to the present model, the possibility that there are different logogens for different cases of a single letter has also been considered. This hypothesis would allow the present data to be reconciled with the traditional notions of a logogen system with variation in the levels of activation of the logogens, but it provides no account of many other findings involving different cases of a letter. A variety of evidence cited in the introduction, as well as a number of subsidiary findings from the present studies, suggests that letter identification generally results in the name of the letter that was presented and that most tasks (including the one used here) are performed with this name code. It is clear that there is a closer relationship between different-case versions of a single letter than between different letters (e.g., Posner \& Keele, 1967). A model in which a logogen is specific to a given letter in a given case does not provide for such relationships, however. Such a model would require a second, higher level of logogens. The case-specific "sublogogens" would have to feed into letter-name logogens, on which performance is based. This model, however, is a special case of the one we propose. The sublogogens, at which the changes in activation level occur, are entirely consistent with the idea that the changes produced by probability are somewhere between the feature level and the letter-name logogen level.

There are two results from studies with word stimuli that converge with the conclusions drawn from these experiments, suggesting that the notion of route-specific activation may not be limited to logogens for letters. First, McClelland (1977) had subjects learn meanings for 16 invented words and practice categorizing these words on the basis of their meanings. During learning and practice, each word appeared in one type font. In a later test phase, words were presented in a very different font. Initial categorizations of words in the new font were somewhat slower than the control, indicating the importance of practice with a specific method of activating the logogens for the invented words. Second, Scarborough, Gerard, and Cortese (1979) studied the effects of repeating words in a pronounciation task. They found that responses were faster to words that had been presented earlier in the experiment, even if the task was changed from pronunciation to recognition. However, facilitation for repeated words was obtained only when the form of presentation was the same on both occasions. Presentation of pictorial stimuli requiring word responses did not facilitate later recognition of the same words. On the other hand, Scarborough, Cortese, and Scarborough (1977) reported that the advantage for repeated words was unaffected by whether the word appeared in the same case when it was repeated. This finding is superficially inconsistent with the idea that repetition produces route-specific facilitation, but a closer analysis suggests a reconciliation. Many theorists conceive of word recognition as a process with at least two distinct steps. An initial step consists of activation along routes from visual features to letter (or lettercluster) logogens, and a later step consists of activation along routes from letter logogens to word logogens (cf. Gough, 1972; McClelland \& Johnston, 1977; Smith \& Spoehr, 1974; Thompson \& Massaro, 1973). If letter logogens are case-independent, as argued earlier, then the second half of the route by which letters activate a word would be the same regardless of the case in which the word is presented. Repetition effects could indeed be route-specific if the facilitation were in the second step of the word activation process. In other words, routespecific facilitation of words could be found along the route by which letter logogens activate word logogens, rather than along the route by which visual features activate letters. Some support for this argument comes from the finding of very weak and short-lived repetition effects for nonwords (Scarborough et al., 1977). Processing to the letter level should be the same for words and nonwords, so a repetition effect in the activation of letter logogens should be the same for words and nonwords. However, a weak repetition effect for nonwords 
is easily explained if the repetition effect occurs in the activation of the word logogens.

Finally, the present results are also diagnostic with respect to certain models of the memory scanning task. An important implication of the results of the present experiments is that stimulus probability has all of its effect before the point at which the name of the stimulus is activated and used. Since the processing for the two different-case versions of a letter is the same past the point at which the name code is activated, a probability effect in these processes would have been revealed by transfer to the other case. Given that there was no transfer, it seems reasonable to conclude that there is no effect of probability on the processes sub. sequent to name identification, at least in the range of probabilities used. It should be noted that the latter qualification is slightly more important than it would appear at first. This is because variations in probability of different letter names were not as extreme as variations in probability of different visual forms. Consider Experiment 1 , for example. The most and least frequent visual forms occurred 85 and 5 times, respectively, whereas the most and least frequent names occurred 90 and 10 times, respectively. Thus the ratio of highest to lowest frequency was $17: 1$ for visual forms, but only $9: 1$ for names. Still the frequency imbalance for names was fairly large, and the failure to find a transfer of probability effect must be considered evidence that probability has all of its effect during encoding in this task.

Because of the requirement that all probability effects occur prior to memory scanning, the present data are inconsistent with the memory scanning model proposed by Theios et al. (1973). In their model, the effect of high probability is to move an item up in a dynamic memory stack. Probe items are compared with items held in the memory stack, and high-probability probes will thus be located in the stack more quickly than will low-probability probes. In this model, response time is essentially determined by the time needed to locate the probe in the stack, so this model has a natural explanation for stimulus probability effects. However, the Theios et al. model involves the use of name codes to search the memory stack and so would be subject to the prediction that the probability effect should transfer in the present experiment. This model is inconsistent with the present results because it places all or almost all of the probability effect after the encoding stage, whereas the present results suggest there is little or no probability effect subsequent to encoding.

The conclusion that stimulus probability has an effect early in visual processing is not a new one. As noted earlier, several studies have reached the same conclusion on the basis of other experimental manipulations (e.g., Geller, 1977; Miller \& Pachella, 1973). The present studies are suggestive of rather specific mechanisms by which this effect may come about, however. Findings of specificity of the stimulus probability effect indicate that the aspects of visual processing sensitive to probability involve both visual and name information. It thus seems very plausible at this point that probability may have its effect on the processes that interpret the visual evidence in order to get the name of the stimulus presented.

The focus on perceptual processes in these experiments should not be taken to imply that probability has effects only on perceptual mechanisms. Within the larger context of the entire information processing system, stimulus probability is emerging as a variable whose influence is seen at many stages. It has been shown that probability has a larger effect when stimulus-response compatibility is low, for example, indicating an effect of probability on some phase of response selection as well as on perceptual mechanisms (Hawkins et al., 1973; Spector \& Lyons, 1976). In view of this diversity of established probability effects, it seems inevitable that any complete model for probability effects must address multiple processing stages. There has been some tendency to try to localize "the effect" of stimulus probability in one stage or another, but it is now apparent that this approach is overly simplistic. While it will undoubtedly be more difficult to design studies acknowledging the diversity of probability effects, it seems obvious that in the long run this is the only promising approach to understanding the phenomena.

\section{REFERENCE NOTES}

1. Wattenbarger, B. The representation of the stimulus in character classification (Tech. Rep. 22). Ann Arbor: University of Michigan, Human Performance Center, 1970.

2. Theios, J. The locus of cognition. Paper presented at the 13th annual meeting of the Psychonomic Society, St. Louis, Missouri, November 1972.

3. Rumelhart, D. A multicomponent theory of confusion among briefly exposed alphabetic characters (Rep. 22). San Diego: University of California, Center for Human Information Processing, 1971.

\section{REFERENCES}

Atkinson, R. C., \& Juola, J. F. Factors influencing speed and accuracy of word recognition. In S. Kornblum (Ed.), Attention and performance IV. New York: Academic Press, 1973.

Besner, D. Character classification: Levels of processing and the effects of stimulus probability. Bulletin of the Psychonomic Society, 1977, 9, 337-339.

Chase, W., \& Calfee, R. Modality and similarity effects in short-term recognition memory. Journal of Experimental Psychology, 1969, 81, 510-514.

Conrad, R. Acoustic confusion and immediate memory. British Journal of Psychology, 1964, 55, 75-84.

Dainoff, M. J. Time course of visual and auditory encoding. Journal of Experimental Psychology, 1970, 86, 214-224.

Geller, E. Latencies to name one of three stimulus dimensions: A study of probability effects and dimension integrality. Perception \& Psychophysics, 1977, 22, 70-76.

Geller, E., Whitman, C., \& Post, D. Stimulus probability and prediction outcome as determinant of choice reaction time: Some procedural considerations. Acta Psychologica, 1973, 37, $1-14$.

Goucir, P. One second of reading. In J. Kavanagh \& I. Mattingly 
(Eds.), Language by ear and eye. Cambridge, Mass: M.I.T. Press, 1972.

Hardzinski, M., \& Pachella, R. The manipulation of visual quality and the definition of stimulus encoding operations in memory scanning experiments. Perception \& Psychophysics, $1980,27,232-240$.

Hawkins, H., Mackay, S., Holley, S., Friedin, B., \& Conen, S. Locus of the relative frequency effect in choice reaction time. Journal of Experimental Psychology, 1973, 101, 90-99.

KeEle, S. W. Attention and human performance. Pacific Palisades, Calif: Goodyear, 1973.

Massaro, D. Experimental psychology and information processing. Chicago: Rand McNally, 1975.

McClelland, J. Letter and configuration information in word identification. Journal of Verbal Learning and Verbal Behavior, 1977, 16, 137-150.

McClelland, J., \& Johnston, J. The role of familiar units in perception of words and nonwords. Perception \& Psychophysics, 1977, 22, 249-261.

Mille R, J. Cognitive influences on perceptual processes. Journal of Experimental Psychology: Human Perception and Performance, $1979,5,546-562$.

Miller, J., \& Pachella, R. On the locus of the stimulus probability effect. Journal of Experimental Psychology, 1973, 101, 227-231.

Miller, J., \& Pachella, R. Encoding processes in memory scanning tasks. Memory \& Cognition, 1976, 4, 501-506.

Morton, J. Interaction of information in word recognition. Psychological Review, 1969, 76, 165-178.

Neisser, U. Cognitive psychology. New York: Appleton-CenturyCrofts, 1967.

PACHELLA, $R$. The interpretation of reaction time in information processing research. In B. Kantowitz (Ed.), Human information processing: Tutorials in performance and cognition. Hillsdale, N.J: Erlbaum, 1974.

Pachella, R., \& Miller, J. Stimulus probability and samedifferent classification. Perception \& Psychophysics, 1976, 19, 29-34.

Posner, M., Boies, S., Eichelman, W., \& Taylor, R. Retention of visual and name codes of single letters. Journal of Experimental Psychology, 1969, 79(1, Pt. 2).

Posner, M., \& Keele, S. W. Decay of visual information from a single letter. Science, 1967, 158, 137-139.

Posner, M., \& Snyder, C. Facilitation and inhibition in the processing of signals. In P. M. A. Rabbitt \& S. Dornic (Eds.), Attention and performance $V$. New York: Academic Press, 1975.

Rumelhart, D. E. A multicomponent theory of the perception of briefly exposed displays. Journal of Mathematical Psychology, 1970, 7, 191-218.

Scarborough, D., Cortese, C., \& Scarborough, H. Frequency and repetition effects in lexical memory. Journal of Experimental Psychology: Human Perception and Performance, 1977 , 3. 1-77.

Scarborough, D., Gerard, L., \& Cortese, C. Accessing lexical memory: The transfer of word repetition effects across task and modality. Memory \& Cognition, 1979, 7, 3-12.

Seamon, J., \& Wright, C. Generative processes in character classification: Evidence for a probe encoding set. Memory \& Cognition, 1976, 4, 96-102.

SIPLE, P. Auditory and visual representation in short-term recognition memory. Unpublished doctoral dissertation, University of California at San Diego, 1975.

Sмiтh, E., \& SPOEнR, K. The perception of printed English: A theoretical perspective. In B. Kantowitz (Ed.), Human informa- tion processing: Tutorials in performance and cognition. Potomac, Md: Erlbaum, 1974.

Spector, A., \& Lyons, R. The locus of stimulus probability effect in choice reaction time. Bulletin of the Psychonomic Society, 1976, 7, 519-521.

Sperling, G. Successive approximations to a model for shortterm memory. Acta Psychologica, 1967, 27, 285-292.

Stanovich, K., \& Pachella, R. The effect of stimulus probability on the speed and accuracy of naming alphanumeric stimuli. Bulletin of the Psychonomic Society, 1976, 8, 281-284.

Sternberg, S. Memory scanning: Mental processes revealed by reaction-time experiments. American Scientist, 1969, 57, 421-457.

The ios, J., Smith, P., Haviland, S., Traupmann, J., \& Moy, M. Memory scanning as a serial, self-terminating process. Journal of Experimental Psychology, 1973, 97, 323-336.

Thompson, M., \& Massaro, D. Visual information and redundancy in reading. Journal of Experimental Psychology, 1973, 98, 49-54.

Turvey, M. On peripheral and central processes in vision: Inferences from an information processing analysis of masking with patterned stimuli. Psychological Review, 1973, 80, 1-52.

Wickelgren, W. A. Acoustic similarity and intrusion errors in short-term memory. Journal of Experimental Psychology, 1965 , 70, 102-108.

\section{NOTES}

1. Geller (1977) reached a different conclusion about the effect of probability on feature detectors. He had subjects name the location, color, or shape of visually presented forms. It took longer to name shape or color when a form was presented in the less probable location than when it was presented in the more probable location, even though location was irrelevant to the decision. Similarly, it took longer to name location or shape when a form was presented in the less probable color than when it was presented in the more probable color. On the basis of these results, Geller concluded that probability influences the speed with which feature detectors operate. He argued that any effect of probability for an irrelevant stimulus dimension must occur during the activation of feature detectors for that dimension, since the dimension is not used in choosing a response. A plausible alternative hypothesis, consistent with the results of Miller (1979), is that subjects can differentially allocate attention to different locations and different colors. Thus naming shape may be faster for a stimulus in a more probable location because more attention is directed to that location at the start of the trial.

2. Pilot data were collected from 17 subjects in order to get an idea of how different the triangular font was from the Rumelhart (Note 3) font. Subjects performed a sequential "same-different" task, and they were instructed to regard different versions (fonts) of the same letter as "different." Subjects averaged $84 \mathrm{msec}$ faster to respond "different" when a Rumelhart letter was followed by the same letter in the triangular font than to respond "different" when a Rumelhart letter was followed by the visually similar Rumelhart letter (566 vs. $650 \mathrm{msec} ; \mathrm{p}<.01)$. A $95 \%$ confidence interval for the size of this advantage included values from 60 to $108 \mathrm{msec}$. Thus, it seems clear that the triangular letters were quite different from the Rumelhart letters, relative to the differences among the Rumelhart letters themselves.

(Received for publication December 27, 1979; revision accepted July 14,1980 .) 